\title{
Dipolar Relaxation of ${ }^{7} \mathrm{Li}$ by Hindered Rotators $\mathrm{ND}_{\mathrm{x}} \mathrm{H}_{3-\mathrm{x}}$ in Lithimn Hydrazinium Sulfate
}

\section{Author: Robert S. Parker \& V. Hugo Schmidt}

NOTICE: this is the author's version of a work that was accepted for publication in Journal of Magnetic Resonance. Changes resulting from the publishing process, such as peer review, editing, corrections, structural formatting, and other quality control mechanisms may not be reflected in this document. Changes may have been made to this work since it was submitted for publication. A definitive version was subsequently published in Journal of Magnetic Resonance, [VOL\# 6, ISSUE\# 4, (1969)]

Parker, Robert S, and V Hugo Schmidt. "Dipolar Relaxation of 7Li by Hindered Rotators NDxH3-x in Lithimn Hydrazinium Sulfate." Journal of Magnetic Resonance (1969) 6, no. 4 (April 1972): 507-515. http://dx.doi.org/10.1016/0022-2364(72)90160-6

Made available through Montana State University's $\underline{\text { ScholarWorks }}$ scholarworks. montana.edu 


\title{
Dipolar Relaxation of ${ }^{7} \mathrm{Li}$ by Hindered Rotators $\mathrm{ND}_{x} \mathrm{H}_{3-x}$ in Lithium Hydrazinium Sulfate ${ }^{*}$
}

\author{
ROBERT S. PARKer

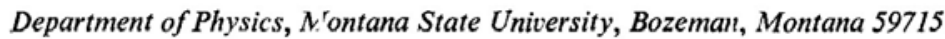 \\ AND \\ V. Hugo SCHMIDT $\dagger$ \\ Laboratory of Solid Stute Physics, E.T.H. CH-8049 Zuri:h, Switzerland \\ Presented at the Fourth international Symposium on Mąnetic Resonance, \\ Israel, August, 1971
}

\begin{abstract}
When a hindered rotation does not change the spatial chirge distribution but merely permutes the locations of nuclear magnetic moments, the resulting spinlattice relaxation of a nearby fixed nucleus is likely to be dipolir even if this nucleus has a quadrupole moment. The dipolar relaxation rate is proportional to the average of $\gamma^{2} I(I+1)$ for the rotating nuclei and accordingly varies lin aarly with percentage of deuteration. For ${ }^{7} \mathrm{Li}$ in $\mathrm{LiN}_{2} \mathrm{H}_{5} \mathrm{SO}_{4}$ at $14 \mathrm{MHz}$ the peak $1 / T$, is $1.8 \mathrm{sec}^{-1}$ at $185^{\circ} \mathrm{K}$, while in $81 \%$ deuterated $\mathrm{LiN}_{2} \mathrm{D}_{5} \mathrm{SO}_{4}$ it is 4.2 times smaller, a: predicted. The $\mathrm{ND}_{2}$ group deuterons also have a $1 / T_{1}$ peak at this temperature, ancl all three $1 / T_{1} v s .1 / T$ curves show activation energy of about $0.19 \mathrm{eV}$, as found previously for protons and deuterons within rotating $\mathrm{NH}_{3}$ and $\mathrm{ND}_{3}$ groups.
\end{abstract}

\section{INTRODUCTION}

Lithium hydrazinium sulfate, $\mathrm{LiN}_{2} \mathrm{H}_{5} \mathrm{SO}_{4}$, has attracted interest because of its unusual dielectric properties. Pepinsky, Vedam, Okaya, and Hoshino (l) observed hysteresis loops for alternating electric fields applied along $c$. Vanderkooy, Cuthbert, and Petch (2) found very anisotropic electrical conductivity, with unusually large protonic conductivity along the $c$ axis, which is parallel to the channels containing the hydrazinium ions. The dielectric susceptibility has very large real and lossy components over a wide frequency and temperature range. Recent experimental results rule out ferroelectricity, and the dielectric properties are explained in terms of nearly onedimensional conduction along the channels and the effect on this conduction of defects which partially block these channels (3).

NMR experiments undertaken to explain the behavior of t is material have displayed a rich variety of phenomena. Petch and collaborators have studied the ${ }^{7} \mathrm{Li}$ spectra and proton line width (4), as well as proton $T_{1}(5)$ and $T_{1 \rho}(6)$, in $\mathrm{LiN}_{2} \mathrm{H}_{5} \mathrm{SO}_{4}$. We have studied the NMR spectra (7) and relaxation (8) for the deuteron sites in $\mathrm{LiN}_{2} \mathrm{D}_{5} \mathrm{SO}_{4}$

* Work supported by National Science Foundation.

$\dagger$ On sabbatical leave from Montana State University. 
previously, and have recently obtained further deuteron results in addition to ${ }^{7} \mathrm{Li}$ spectra and $T_{1}$ data in both normal and deuterated crystals.

Quite similar processes occur in both crystals. The ${ }^{7} \mathrm{Li}$ spectra indicate a hightemperature structural change of undetermined nature. Proton line width and $T_{1 \rho}$ measurements indicate $\mathrm{N}_{2} \mathrm{H}_{5}^{+}$motion (with activation energy of $0.69 \mathrm{eV}$ ), and such motion may explain the observed mixing of $\mathrm{ND}_{2}$ and $\mathrm{ND}_{3}^{+}$populations $(0.75 \mathrm{eV})$. Proton line width, $T_{1}$ and $T_{1 \rho}$ measurements as well as deuteron spectral line merging and $T_{1}$ results indicate hindered rotations of $\mathrm{NH}_{2}(0.46 \mathrm{eV}), \mathrm{ND}_{2}(0.50 \mathrm{eV}), \mathrm{NH}_{3}^{+}$ $(0.19 \mathrm{eV})$, and $\mathrm{ND}_{3}^{+}(0.20 \mathrm{eV})$ groups. The NMR measurements show that these hindered rotations involve permutations of the two (three) hydrogens among their two (three) possible sites $(6,7)$.

Important for this study are the $\mathrm{NH}_{3}^{+}$and $\mathrm{ND}_{3}^{+}$hindered rotations, because they give $T_{1}$ minima near $185^{\circ} \mathrm{K}$ for ordinary NMR frequencies. This is far below the decomposition temperature and in a region in which other relaxation mechanisms are weak, so that effects of this motion can be easily studied over a wide temperature range. The $T_{1}$ minima are $20 \mathrm{msec}$ for protons at $27 \mathrm{MHz}(5)$ and $0.8 \mathrm{msec}$ for deuterons at $14 \mathrm{MHz}$ (8). The deuteron $T_{1}$ is shorter because the quadrupolar interaction energy fluctuations resulting from different electric field gradients (efg's) at the different deuteron sites are large compared to the proton dipolar energy fluctuations.

For a nearby nucleus not partaking in the hindered rotation, the situation is different. The hindered rotation causes no charge redistribution and hence no efg change at the fixed nucleus except for the extremely short interval during which the rotation occurs. The rotation does permute the positions of the three nuclear magnetic moments, causing magnetic field fluctuations resulting in dipolar relaxation for the nearby fixed nucleus.

The theory for this dipolar relaxation is developed in Section II. Experimental results for $\mathrm{ND}_{2}$ deuteron and ${ }^{7} \mathrm{Li} T_{1}$ appear in Section III and are compared with theory in Section IV.

\section{THEORY}

The starting point is the equation

$$
1 / T_{1}=\gamma_{\mathrm{N}}^{2}\left(\overline{H_{x}^{2}}+\overline{H_{y}^{2}}-\bar{H}_{x}^{2}-\bar{H}_{y}^{2}\right) \tau_{0} /\left(1+\omega^{2} \tau_{0}^{2}\right)
$$

which is derived by Slichter (9) for nuclear spins which couple to magnetic field fluctuations but not to each other. However, in order to obtain a single relaxation time $T_{1}$ as is experimentally observed, we assume interactions between nuclei to be strong enough to maintain a common spin temperature throughout the spin-lattice relaxation process. This equation is valid for nuclei having arbitrary spin $I$, if the applied field $H_{0}$ is large enough so that the Zeeman energy difference $\gamma_{N} \hbar H_{0}=\hbar \omega$ between adjacent levels is large compared to the quadrupolar interaction energy.

The form of Eq. [1] results from assuming a single correlation time $\tau_{0}$ for the field seen at the fixed nucleus. This assumption is shown to be correct for our case in the following calculation. We consider the autocorrelation function $\overline{H(0) H(\tau)}$ for a component of this field, which can assume three values $H_{i}$ corresponding to the three positions $\left(0^{\circ}, \pm 120^{\circ}\right)$ of the $\mathrm{ND}_{x} \mathrm{H}_{3-x}$ hindered rotator. Let $n_{i j}(\tau)$ be the probability that 
a rotator initially in the $i$-th position will be in the $j$-th position after a time interval $\tau$. This probability is derived from the differential equation

$$
d n_{i} / d_{i}=-n_{i} / \tau_{r}+\frac{1}{2}\left(1-n_{i}\right) / \tau_{r}
$$

for the probability $n_{i}$ that a rotator is in the $i$-th position. Here $\tau_{r}$ is the mean time between random $\pm 120^{\circ}$ rotations and $\frac{1}{2}$ is the probability that: a rotator in a position $j \neq i$ will return to the $i$-th position with the next rotation. Thi:s equation gives

$$
n_{i j}(\tau)=\pi+\left(\delta_{i j}-\frac{1}{3}\right) e^{-\tau / \tau_{0}} ; \tau_{0}=\frac{2}{3} \tau_{r} .
$$

The autocorrelation function ther becomes

$$
\begin{aligned}
\overline{H(0) H(\tau)} & =\frac{1}{3} \sum_{i, j} H_{i} H_{j} n_{i j}(\tau) \\
& =\bar{H}^{2}+\frac{1}{9} \sum_{i, j}^{\prime}\left(H_{i}-H_{j}\right)^{2} e^{-\tau / \tau},
\end{aligned}
$$

where the prime indicates $i<j$. The term $\bar{H}^{2}$ does not contribute to the Fourier transform and accordingly such terms must be subtracted from the autocorrelation function for $\tau=0$, as is done in Eq. [1]. Slichter's expression omits these $\bar{H}_{x}^{2}$ and $\bar{H}_{y}^{2}$ terms, which are zero for his example. We must retain them, because $H_{x}$ and $H_{y}$ c a time long compared to $T_{1}$ for the nuclei in the rotating grcups, and this $T_{1}$ is long compared to $\tau_{0}$.

Slichter assumes that $\overline{H_{x}(0) H_{y}(0)}=0$ in his derivation. If this term is nonzero, it can be made to equal zero by a suitable rotation of the $x$ and $y$ axes. Since the term $\overline{H_{x}^{2}}+{\overline{H_{y}^{2}}}_{-}-\bar{H}_{x}^{2}-\bar{H}_{y}^{2}$ in Eq. [1] is invariant with respect to this axis rotation, it follows that the term $\overline{H_{x}(0) H_{y}(0)}$ does not affect $T_{1}$.

To calculate the fields at a fixed nucleus, we note that a rotating group has three nuclei which permute positions among three sites when hincered rotation occurs. A dipole $\mu_{j}$ aligned with $H_{0}$ and located at the $i$-th site will crecte at the fixed nucleus a field having a component in the $x-y$ plane of magnitude $3 \mu_{j} \sin 2 \theta_{i} / 2 r_{i}^{3} \equiv \mu_{j} h_{i}$, where $\theta_{i}$ and $r_{i}$ are the polar angle and length of a vector from site $i$ tis the fixed nucleus. Then if $\phi_{i}$ is the corresponding azimuthal angle and $k$ is an index for the three positions of the rotating group, the average field at the fixed nucleus has an $x$ component

$$
\overline{H_{x}}=\frac{1}{3} \sum_{i, k} \mu_{i+k} h_{i} \cos \phi_{i}=\bar{\mu} \sum_{i} h_{i} \cos \phi_{i}
$$

using $\bar{\mu} \equiv \frac{1}{3} \sum_{j} \mu_{j}$. Here $i+k$ is a cyclic index having values 1,2 , or 3 ; so, for the example, $i=k=2, i+k=4-3=1$. The autocorrelation function for $H_{x}$ at zero time is

$$
\begin{aligned}
\overline{H_{x}^{2}} & =\frac{1}{3} \sum_{i, j, k} \mu_{i+k} \mu_{j+k} h_{i} h_{j} \cos \phi_{i} \cos \phi_{j} \\
& =\frac{1}{3} \sum_{i, j}\left(3 \bar{\mu}^{2}+\sum_{k} \Delta_{i+k} \Delta_{j+k}\right) h_{i} h_{j} \cos \phi_{i} \cos \phi_{j} \\
& =\bar{H}_{x}^{2}+\overline{\Delta^{2}} \sum_{i} h_{i}^{2} \cos ^{2} \phi_{i}-\overline{\Delta^{2}} \sum_{i, j}^{\prime} h_{i} h_{j} \cos \phi_{i} \cos \phi_{j} \\
& =\bar{H}_{x}^{2}\left(1-\overline{\Delta^{2}} / 2 \bar{\mu}^{2}\right)+\frac{3}{2} \overline{\Delta^{2}} \sum h_{i}^{2} \cos ^{2} \phi_{i},
\end{aligned}
$$


using the definitions $\Delta_{i} \equiv \mu_{i}-\bar{\mu}$ and $\overline{\Delta^{2}} \equiv \frac{1}{3} \sum_{i} \Delta_{i}^{2}$, noting that $\frac{Y}{i} \Delta_{i}=0$, and using $\sum_{i} \Delta_{i} \sum_{j} \Delta_{j}=0$ to transform the second expression. For $H_{y}$, Eqs. [2] and [3] have $\sin \phi$ in place of $\cos \phi$, so that

$$
\begin{aligned}
\bar{H}_{x}^{2}+\bar{H}_{y}^{2}-\bar{H}_{x}^{2}-\bar{H}_{y}^{2} & =\frac{3}{2} \overline{\Delta^{2}} \sum_{i} h_{i}^{2}-\left(\bar{H}_{x}^{2}+\bar{H}_{y}^{2}\right) \overline{\Delta^{2}} / 2 \bar{\mu}^{2} \\
& =\frac{1}{2} \overline{\Delta^{2}} \sum_{i, j}^{\prime}\left[h_{i}^{2}+h_{j}^{2}-2 h_{i} h_{j} \cos \left(\phi_{i}-\phi_{j}\right)\right] .
\end{aligned}
$$

This is the magnetic field factor in Eq. [1], which is independent of choice of $x$ and $y$ axes as expected. The summand in the final expression is the square of the difference of vectors of lengths $h_{i}$ and $h_{j}$ separated by an angle $\phi_{i}-\phi_{j}$. The corresponding sum can be called the geometrical factor in $1 / T_{1}$, as it contains only spatial quantities, and is the only factor which contains them.

We now evaluate the factor $\overline{\Delta^{2}}$. Up to this point the average has been only over the three nuclei of one particular hindered rotator. With the assumption that mutual spin flips maintain a common spin temperature for the relaxing nuclei at all times, the calculation of the common $1 / T_{1}$ for these nuclei requires that the average must be taken also over all rotators. First, the average for one rotator can be expressed as

$$
\overline{\Delta^{2}}\left(\mu_{i}\right)=\frac{1}{3} \sum_{i}\left(\mu_{i}-\bar{\mu}\right)^{2}=\frac{1}{9} \hbar^{2} \sum_{i, j}^{\prime}\left(\gamma_{i} m_{i}-\gamma_{j} m_{j}\right)^{2} .
$$

Then the average is taken for all rotators of a given isotopic composition $\mathrm{ND}_{x} \mathrm{H}_{3-x}$, where $x$ is an integer. At all temperatures of interest it is an extremely good approximation to assume equal probability for each set of three $m$ values, so that the cross terms in Eq. [8] vanish in this average, which then becomes

$$
\begin{aligned}
\overline{\Delta^{2}}(x) & =\frac{2 \hbar^{2}}{9} \sum_{m_{1}} \sum_{m_{2}} \sum_{m_{3}} \frac{\gamma_{1}^{2} m_{1}^{2}+\gamma_{2}^{2} m_{2}^{2}+\gamma_{3}^{2} m_{3}^{2}}{\left(2 I_{1}+1\right)\left(2 I_{2}+1\right)\left(2 I_{3}+1\right)} \\
& =\frac{2 \hbar^{2}}{27} \sum_{i} \gamma_{i}^{2} I_{i}\left(I_{i}+1\right)=\frac{2}{27}\left[x \mu_{\mathrm{D}}^{2}+(3-x) \mu_{\mathrm{H}}^{2}\right] .
\end{aligned}
$$

If deuterons randomly occupy a fraction $f$ of the hydrogen sites in the rotating groups, the probability that a given rotator contains $x$ deuterons is $f^{x}(1-f)^{3-x} 3$ !/ $x !(3-x)$ !. The sum from $x=0$ to $x=3$ of the products of $x$ with these weighting factors is simply $3 f$, so the mean square magnetic moment deviation averaged over all rotators is

$$
\overline{\Delta^{2}}=\frac{2}{9}\left[f \mu_{\mathrm{D}}^{2}+(1-f) \mu_{\mathrm{H}}^{2}\right]=\overline{\frac{2}{9} \mu_{\mathrm{hyd}}^{2}} .
$$

Accordingly, $1 / T_{1}$ is proportional to the mean square magnetic moment of the hydrogen nuclei averaged over all of the rotating groups, and thus varies linearly with percentage of deuteration.

Putting all the factors together, we obtain

$$
1 / T_{1}=\gamma_{\mathrm{N}}^{2} \overline{\mu_{\mathrm{hyd}}^{2}} G \tau_{0} /\left(1+\omega^{2} \tau_{0}^{2}\right),
$$


where $\mu^{2} \equiv \gamma^{2} \hbar^{2} I(I+1)$ is averag sd over all hydrogens, and in which the complete geometrical factor $G$ is given by

$$
G=\frac{1}{4} \sum_{L} \sum_{i, j}^{\prime}\left[\frac{\sin ^{2} 2 \theta_{i}}{r_{i}^{6}}-\frac{\sin ^{2} 2 \theta_{j}}{r_{j}^{6}}-\frac{2 \sin 2 \theta_{i} \sin 2 \theta_{j}}{r_{i}^{3} r_{j}^{3}} \cos \left(\phi_{i}-\phi_{j}\right)\right],
$$

with $\sum_{L}$ representing a lattice sum over all rotating groups.

This theory is also applicable to dipolar relaxation caused by groups containing two magnetic moments which exchangre positions when hindered totation occurs, such as $\mathrm{H}_{2} \mathrm{O}$ or $\mathrm{NH}_{2}$ groups in certain crystals. Only certain numer cal factors change; the most important of these changes a.re now described. The $\frac{1}{2}$ disappears from Eq. [2], so that $\tau_{0}=\frac{1}{2} \tau_{r}$. The geometrical facior in the last line of Eq. [7] also loses a factor $\frac{1}{2}$, and the primed sum contains only onc: term. In Eq. [10] the coefficient of $\overline{\mu_{\text {hyd }}^{2}}$ becomes $\frac{1}{6}$, and the coefficient of $G$ in Eq. [12] changes to $\frac{3}{8}$. The expression for $1 / T_{1}$ in Eq. [11] remains unchanged except for these redefinitions of $G$ and $\tau_{0}$.

\section{EXPERIMENTAL}

Crystals were grown by evaporation from a water solution of $\mathrm{Li}_{2} \mathrm{CO}_{3}$ and $\mathrm{N}_{2} \mathrm{H}_{4} \cdot \mathrm{H}_{2} \mathrm{SO}_{4}$. Deuterated crystals were recrystallized twice fom $\mathrm{D}_{2} \mathrm{O}$, and the final crystals were found by mass spectrometry to contain 81 atomic percent deuterons $(10)$.

The $T_{1}$ measurements were made at $14 \mathrm{MHz}$. The temperature was regulated by a feedback control system, with boil-off from liquid nitrogen us:ed as a coolant. For $T_{1}$ longer than two minutes, a modified Robinson (11) NMR spectrometer was used, while for shorter $T_{1}$ a pulse NMR circuit of Clark's (12) design wa; employed. With either apparatus the experimental sequence was saturation followed after an interval $t$ by a pulse or rapid sweep to observe the recovery.

All measurements were made with applied field $H_{0}$ parallel to the crystalline $b$ axis. (Here the notation of Brown's (13) X-ray investigation is used; Padmanabhan and Balasubramanian (14) in their neutron diffraction study interchanged the $a$ and $b$ axes.) For this orientation all molecules become physically as well as chemically equivalent, so the ${ }^{7} \mathrm{Li}$ spectrum contains only three lines. Below room temperature, the $\mathrm{ND}_{2}$ deuteron spectrum contains four lines, two from $\mathrm{D}(1)$ which is i a weak $\mathrm{N}-\mathrm{D} \cdots \mathrm{O}$ bond and two from $\mathrm{D}(2)$ which is in the $\mathrm{N}-\mathrm{D} \cdots \mathrm{N}$ bond which joins adjacent hydrazinium ions. Above room temperature the rapid $\mathrm{ND}_{2}$ hindered rotaticn merges these lines to a single pair in this orientation (7).

\section{DISCUSSION}

The ${ }^{7} \mathrm{Li}$ measurements were made by saturation of the entire spectrum, followed by later observation of one of the satellite lines. The recovery of its signal height was characterized by a single time constant $T_{1}$. Because different ${ }^{7} \mathrm{Li}$ sites have $\mathrm{ND}_{x} \mathrm{H}_{3-x}$ neighbors with different values of $\sum_{i} \Delta_{i}^{2}$, one would expect a distribution of $T_{1}$ 's. Accordingly, we assume that mutual spin flips maintain a common spin temperature for the ${ }^{7} \mathrm{Li}$ nuclei.

The deuteron $T_{1}$ measurements were made by first saturating one of the two lines from the $\mathrm{D}(1)$ sites, then waiting a time $t$ before obtaining the response for this line. The increase of signal height with $t$ for this unsymmetric saturation usually has two time 
constants. The first $\left(P_{1}+2 P_{2}\right)^{-1}$ is designated $T_{1}$ and is the only time constant for symmetric saturation which gives relaxation for which the populations of the $m=0$ and $m= \pm 1$ states have a Boltzmann distribution at all times. The other $\left(3 P_{1}\right)^{-1}$ governs the approach to a Boltzmann distribution. Here $P_{1}$ and $P_{2}$ are the $\Delta m=1$ and $\Delta m=2$ transition probabilities, respectively, and $P_{2}=0$ for the dipolar relaxation caused by hindered rotation of the $\mathrm{ND}_{x} \mathrm{H}_{3-x}$ groups. These time constants are for relaxation of the $\mathrm{D}(1)$ deuterons at temperatures for which the $\mathrm{ND}_{2}$ hindered rotation time $t_{r}$ is large compared to $T_{1}$, while for the opposite case the average over sites (1) and (2) is obtained.

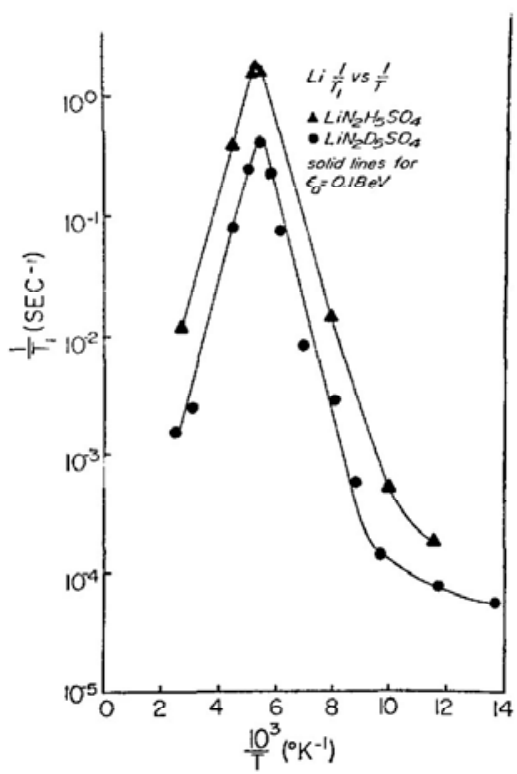

FIG. 1. Temperature dependence of the ' $\mathrm{Li}$ spin-lattice relaxation rate at $14 \mathrm{MHz}$ in $\mathrm{LiN}_{2} \mathrm{H}_{5} \mathrm{SO}_{4}$ and mostly deuterated $\mathrm{LiN}_{2} \mathrm{D}_{5} \mathrm{SO}_{4}$.

For the intermediate case $t_{r} \simeq T_{1}$ there are four time constants, given by expressions derived for the mathematically equivalent situation of interbond jumping in $\mathrm{KD}_{2} \mathrm{PO}_{4}$ (15).

The experimental results are plotted in Fig. 1 for the ${ }^{7} \mathrm{Li}$ relaxation in $\mathrm{LiN}_{2} \mathrm{H}_{5} \mathrm{SO}_{4}$ and mostly deuterated $\mathrm{LiN}_{2} \mathrm{D}_{5} \mathrm{SO}_{4}$, and in Fig. 2 for the longer time constant for the $\mathrm{D}(1)$ relaxation in the latter crystal. The activation energies obtained from the slopes of these curves agree within experimental error with the previously determined activation energies for hindered rotation of the $\mathrm{NH}_{3}^{+}$groups (6) and $\mathrm{ND}_{3}^{+}$groups (8).

At $170^{\circ} \mathrm{K}$ where the $\mathrm{D}(1) 1 / T_{1}$ peak occurs, the $\mathrm{ND}_{2}$ rotation time calculated from $t_{r}=2.2 \times 10^{-14} \mathrm{sec} \exp (0.50 \mathrm{eV} / k T)$ is $10 \mathrm{sec}$. This value is sensitive to errors in the activation energy and preexponential time determined from previous $\mathrm{ND}_{2}$ deuteron $T_{1}$ measurements $(8)$, so that one can only say that $t_{r} \sim T_{1}$ at the $1 / T_{1}$ peak. In light of the previous discussion, it is accordingly not known whether the time constants at the peak are for $\mathrm{D}(1)$ sites, averages over $\mathrm{D}(1)$ and $\mathrm{D}(2)$ sites, or composites of $t_{r}, P_{1}$, and $P_{2}$. 
A comparison is made in Table 1 of experimental peak $1 / T_{1}$ 's with calculated peak values for the dipolar relaxation inechanism. At the peak, thi: factor $\tau_{0} /\left(1+\omega^{2} \tau_{0}^{2}\right)=$ $1 / 2 \omega$ in Eq. [11], so that no uncert ain factors appear in the calculated peak values. For

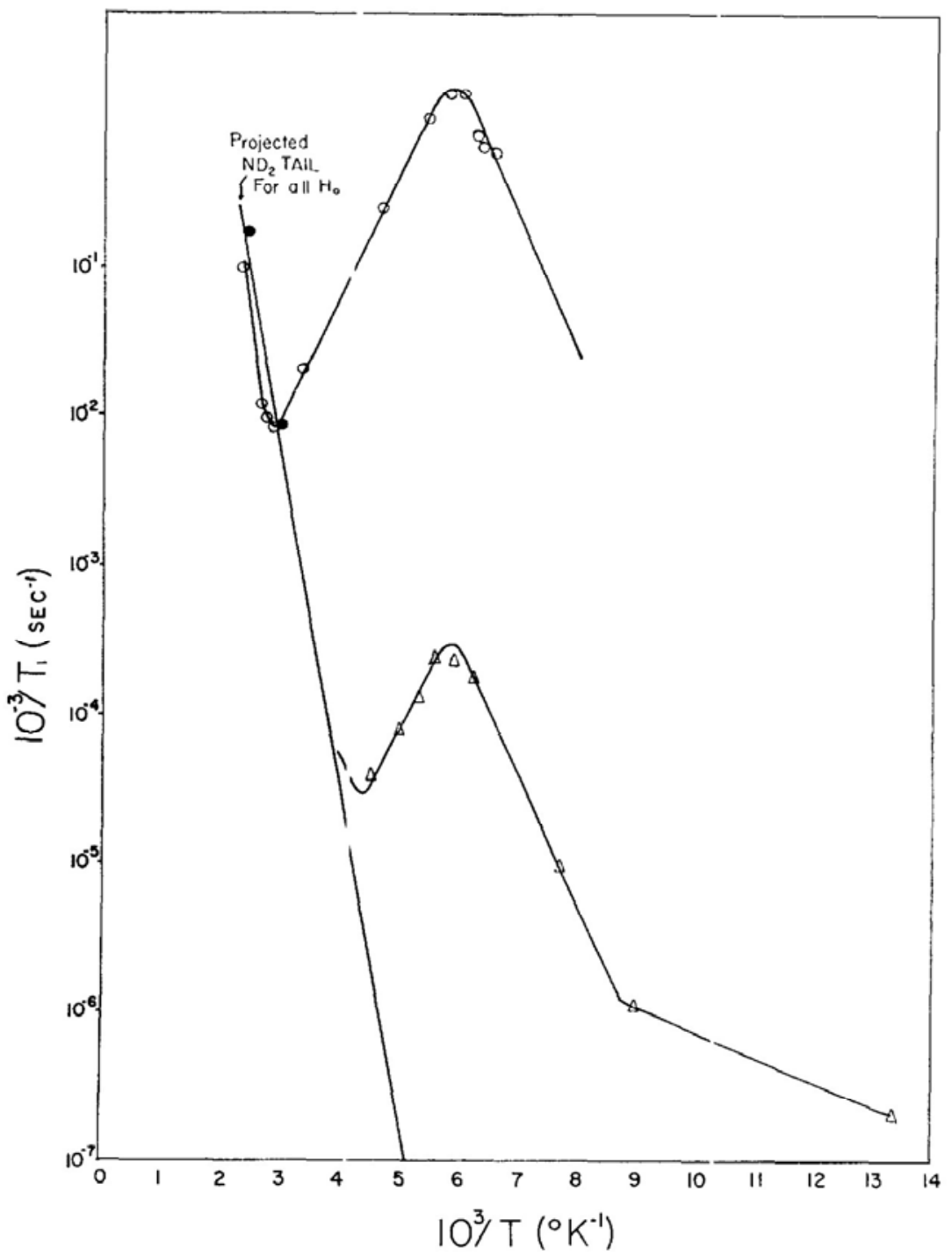

Fig. 2. Temperature dependence of the deuteron spin-lattice relaxation rate at $14 \mathrm{MHz}$ in mostly deuterated $\mathrm{LiN}_{2} \mathrm{D}_{5} \mathrm{SO}_{4}$. Open and filled circles are previous results fcr $\mathrm{ND}_{3}^{+}$and $\mathrm{ND}_{2}$ deuterons respectively [Ref. (8)]. Triangles represent present results for the $\mathrm{D}(1)$ site (to right of peak) and an average over the $\mathrm{D}(1)$ and $\mathrm{D}(2)$ sites (to left of peak), for the slower component of the relaxation.

the ${ }^{7} \mathrm{Li}$ relaxation in both crystals the agreement is nearly w thin experimental error. For $\mathrm{D}(1)$ the calculated relaxation rate is too slow by a factor of two. This factor seems too large to be caused by the uncertainty in the nature of the quantity labeled $T_{1}$. It is 
TABLE 1

Comparison of Measured Peak $1 / T_{1}$ 's with Values

Calculated for Dipolar Relaxation

\begin{tabular}{cccc}
\hline $\begin{array}{c}\text { Percent } \\
\text { deuteration }\end{array}$ & $\begin{array}{c}\text { Nucleus } \\
\text { and site }\end{array}$ & $\begin{array}{c}\text { Peak } 1 / T_{1}\left(\mathrm{sec}^{-1}\right) \\
\text { Calculated } \\
\text { Measured }\end{array}$ \\
\hline 0 & ${ }^{7} \mathrm{Li}$ & 1.350 & 1.84 \\
100 & ${ }^{7} \mathrm{Li}$ & 0.085 & - \\
81 & ${ }^{7} \mathrm{Li}$ & 0.325 & 0.434 \\
81 & $\mathrm{D}(1)$ & 0.114 & $0.250^{a}$ \\
81 & $\mathrm{D}(2)$ & 0.019 & - \\
\hline
\end{tabular}

${ }^{a}$ See discussion in text.

possible that quadrupolar relaxation occurs in this mixed crystal because of slightly different dimensions for bonds containing deuterons. Such differences cause a different efg tensor for ${ }^{7} \mathrm{Li}$ in the deuterated crystal (16) compared to the normal crystal (4). If the efg fluctuations at $\mathrm{D}(1)$ and $\mathrm{D}(2)$ caused by hindered rotation of $\mathrm{ND}_{2} \mathrm{H}^{+}$and $\mathrm{NDH}_{2}^{+}$ groups are assumed to have magnitude equal to the change in efg at the ${ }^{7} \mathrm{Li}$ site upon deuteration, an order-of-magnitude calculation gives quadrupolar relaxation of the required magnitude for these deuterons. Another possible cause of this discrepancy is the neglect of the $x$ and $y$ components of the magnetic moments causing the fluctuating fields. These can be important for the $\mathrm{ND}_{2}$ deuteron relaxation because the frequencies of the $\mathrm{ND}_{3}$ and $\mathrm{ND}_{2}$ deuterons differ only because of relatively small quadrupolar splittings. The smaller ${ }^{7} \mathrm{Li} 1 / T_{1}$ discrepancy probably has this latter origin.

In conclusion, it appears that in crystals in which hindered rotation without charge redistribution occurs for rotators such as $\mathrm{H}_{2} \mathrm{O}, \mathrm{NH}_{2}, \mathrm{NH}_{3}^{+}$, and $\mathrm{NH}_{4}^{+}$, the relaxation of nearby fixed nuclei is often dipolar even if these nuclei possess quadrupole moments. Because the peak relaxation rate is exactly calculable and varies linearly with isotopic composition of the rotating group, it is possible to make quantitative determinations of isotopic composition from $T_{1}$ measurements. Because the deuteron's magnetic moment is much smaller than the proton's, this method is particularly sensitive for detecting small percentages of protons in deuterated crystals.

\section{ACKNOWLEDGMENTS}

We wish to thank Dr. F. L. Howell who grew the crystals, and Dr. R. Bärtschi and Mr. F. Rudin who determined their degree of deuteration, and Dr. C. P. Slichter for a helpful discussion.

One of us (VHS) is grateful to the staff of the Swiss Federal Technical University (ETH) for their hospitality.

\section{REFERENCES}

1. R. Pepinsky, K. Vedam, Y. Okaya, and S. Hoshino, Phys. Rev. 111, 1467 (1958).

2. J. Vanderkooy, J. D. Cuthrert, and H. E. Petch, Can. J. Phys. 42, 1871 (1964).

3. V. H. SCHMid, J. E. Drumheller, AND F. L. Howell, Phys. Rev., B4, 4582 (1971).

4. J. D. Cuthrert and H. E. Petch, Can. J. Phys. 41, 1629 (1963).

5. W. D. MacClement, M. M. Pintar, and H. E. Petch, Can. J. Phys. 45, 3257 (1967).

6. R. R. KNISPEL AND H. E. Petch, Can. J. Phys. 49, 870 (1971). 
7. F. L. Howell and V. H. Schmidt, J. Chem. Phys. 51, 1983 (1969).

8. V. H. SChmidt AND F. L. Howell, J. Phys. Soc. Jap. Suppl. 28, 106 (1970).

9. C. P. SLichter, "Principles of Magnet c Resonance,"Section 5.7, Harper \& Row, New York, 1963.

10. R. BÄRTSCHI AND F. RUDIN, private communication.

11. F. N. H. RoBinson, J. Sci. Instr. 36, +181 (1959).

12. W. G. Clark, Rev. Sci. Instr. 35, 316 1964); W. G. Clark and A. L. Kerlin, ibid. 38, 1593 (1967).

13. I. D. BROWN, Acta Crystallogr. 17, 6 ;4 (1964).

14. V. M. Padmanabhan and R. Balasibramanian, Acta Crystallogr. 22, 532 (1967).

15. V. H. Schmidt ANd E. A. Uehling, Phys, Rev. 126, 447 (1962).

16. R. S. Parker and V. H. Schmidt, Bull. Amer. Phys. Soc. 16, 94 (1971); R. S. Parker, thesis, Montana State University, 1971 ( 1 npublished). 